\title{
Age-Related Cutoffs of Plasma Aldosterone/Renin Concentration for Primary Aldosteronism Screening
}

\author{
Linqiang Ma ${ }^{10},{ }^{1}$ Ying Song, ${ }^{1}$ Mei Mei, ${ }^{1}$ Wenwen He, ${ }^{1}$ Jinbo $\mathrm{Hu}^{1}{ }^{1}$ Qingfeng Cheng, \\ Ziwei Tang, ${ }^{1}$ Ting Luo ${ }^{D}$, ${ }^{1}$ Yue Wang, ${ }^{1}$ Qianna Zhen, ${ }^{1}$ Zhihong Wang ${ }^{D},{ }^{1}$ Hua Qing, \\ Yihong He, ${ }^{2}$ Qifu Li $\mathbb{D}^{1},{ }^{1}$ Shumin Yang $\mathbb{D},{ }^{1}$ and the Chongqing Primary Aldosteronism \\ Study (CONPASS) Group ${ }^{1}$
}

${ }^{1}$ Department of Endocrinology, The First Affiliated Hospital of Chongqing Medical University, Chongqing, China

${ }^{2}$ Department of Radiology, The First Affiliated Hospital of Chongqing Medical University, Chongqing, China

Correspondence should be addressed to Qifu Li; liqifu@yeah.net and Shumin Yang; 443068494@qq.com

Received 4 December 2017; Revised 19 March 2018; Accepted 26 April 2018; Published 16 July 2018

Academic Editor: Thomas J. Fahey

Copyright (c) 2018 Linqiang Ma et al. This is an open access article distributed under the Creative Commons Attribution License, which permits unrestricted use, distribution, and reproduction in any medium, provided the original work is properly cited.

\begin{abstract}
Aim. This retrospective study is aimed at investigating whether aldosterone-renin ratio (ARR) cutoffs calculated by the plasma aldosterone concentration (PAC)/plasma renin concentration (PRC) should be set differently in patients of different ages. Methods. 521 hypertensive patients were screened for primary aldosteronism (PA) by the PAC/PRC. 174 patients diagnosed with PA and 311 patients with essential hypertension $(\mathrm{EH})$ were included in the final analysis. Subjects were subdivided into four age groups: $<40,40-49,50-59$, and $\geq 60$ years old. Results. The accuracy of the ARR varied greatly among the different age groups. An ARR of $3.7(\mathrm{ng} / \mathrm{dl}) /(\mu \mathrm{IU} / \mathrm{ml})$ had a sensitivity of $100 \%$ and a specificity of $80 \%$ in patients $\geq 60$ years old. With this cutoff, the sensitivities in patients $<40,40-49$, and 50-59 years old were $74 \%, 82 \%$, and $87 \%$, respectively, and the specificities were $94 \%, 95 \%$, and 94\%, respectively. To achieve a sensitivity higher than $90 \%$, the ARR cutoff needed to be lowered to $2.0(\mathrm{ng} / \mathrm{dl}) /(\mu \mathrm{IU} / \mathrm{ml})$ for patients $40-49$ and 50-59 years old, resulting in sensitivities of $90 \%$ and $95 \%$, respectively, and specificities of $80 \%$ and $84 \%$, respectively. To achieve a sensitivity higher than $90 \%$, the ARR cutoff needed to be lowered to $1.0(\mathrm{ng} / \mathrm{dl}) /(\mu \mathrm{IU} / \mathrm{ml})$ for patients $<40$ years old, resulting in a sensitivity of $90 \%$ and a specificity of $82 \%$. Conclusions. An ARR of $3.7(\mathrm{ng} / \mathrm{dl}) /(\mu \mathrm{IU} / \mathrm{ml})$ is optimal for patients $\geq 60$ years; for patients $40-59$ years, the optimal ARR cutoff is 2.0 ; for those younger than 40 years, an ARR of 1.0 may be more reasonable.
\end{abstract}

\section{Introduction}

Primary aldosteronism (PA) is caused by adrenocortical lesions and is characterized by autonomous secretion of aldosterone. The aldosterone-renin ratio (ARR) is advocated as the most reliable tool for PA screening in high-risk groups of hypertensive patients [1]. However, appropriate ARR cutoffs should be selected cautiously and validated clinically, as the ARR varies substantially depending on the analytical methods used [2]. Conventionally, plasma renin activity (PRA) is measured by radioimmunoassay to calculate the ARR; however, the plasma renin concentration (PRC), which is measured by an automated chemiluminescent immunoassay, has become increasingly popular because this procedure is less laborious and time consuming [3, 4]. Previous studies have revealed that PRA and PRC were well correlated and that the automated renin chemiluminescent assay was a reliable alternative to the radioimmunometric method [5-8]. Our previous meta-analysis that analyzed the accuracy of PAC/PRC as a screening test in patients with PA revealed that the overall sensitivity, specificity, and area under the curve of PAC/PRC were 0.89, 0.96, and 0.985, respectively, demonstrating the efficacy of PAC/PRC as a screening test for PA [9]. Currently, there are no unanimous 
cutoff values for the ARR for PA screening, and the PRCbased ARR cutoffs mentioned in previous studies varied between 1.0-5.7 (ng/dl)/( $\mu \mathrm{IU} / \mathrm{ml})$ [3-8, 10-14].

Globally, the population is aging and the World Health Organization predicts that by 2050, the population aged 60 years or older will double. PA is more common in young or middle-aged hypertensive patients; however, as the population ages, PA screening may become more popular in elderly patients with hypertension. It is generally accepted that both the plasma renin and aldosterone levels tend to fall with advancing age and plasma renin decreases more than aldosterone; consequently, the ARR increases in the elderly [1]. Thus, using the same ARR cutoff in patients of different ages for PA screening might cause false positive results and render unnecessary confirmatory testing, such as saline infusion testing, which carries a potential risk for acute volume overload in elderly patients. Several studies have focused on the effects of age on PA screening but drew inconsistent conclusions. Yin et al. [15] reported that the cutoffs for the ARR for PA screening were not affected by age, while two other studies $[16,17]$ suggested that the ARR criteria might need to be higher with advancing age. However, the ARR in these studies was based on the PRA and few studies have evaluated the influence of aging on the ARR cutoffs calculated with the PRC.

In this retrospective study, ARR cutoffs based on PRC were evaluated in 485 hypertensive patients and whether or not ARR cutoffs should be set differently in patients of different ages for PA screening was investigated.

\section{Methods}

2.1. Participants. This study retrospectively analyzed data from a previously published prospective study that was conducted in the Department of Endocrinology at the First Affiliated Hospital of Chongqing Medical University from November 2013 to September 2016 [18]. The protocol was approved by the ethics committee of the First Affiliated Hospital of Chongqing Medical University. Written informed consent was obtained from each participant. This prospective study is aimed at evaluating the diagnostic accuracy of the saline infusion test and the captopril challenge test, using the fludrocortisone suppression test as the reference standard, in patients with a high risk for PA according to the following criteria: Joint National Commission stage 2 (blood pressure, BP > 160-179/100-109 mm Hg), stage 3 (BP > 180/ $110 \mathrm{~mm} \mathrm{Hg}$ ), or drug-resistant hypertension; hypertension and spontaneous or diuretic-induced hypokalemia; hypertension with adrenal incidentaloma; hypertension and a family history of early-onset hypertension or cerebrovascular accident at a young age ( $<40$ years); or a hypertensive firstdegree relative of PA.

The exclusion criteria were as follows: patients diagnosed with secondary hypertension other than PA and patients who screened positive but failed to complete any confirmatory testing and did not have a conclusive diagnosis. However, patients with spontaneous hypokalemia, undetectable renin concentrations, and a $\mathrm{PAC}>20 \mathrm{ng} / \mathrm{dl}$ were included as PA patients, even though no further confirmatory testing was performed.

2.2. Screening. For screening tests, treatment with diuretics was withdrawn for at least four weeks, and $\beta$-blockers, angiotensin-converting enzyme inhibitors, and angiotensin1 receptor blockers were stopped for at least two weeks. Only nondihydropyridine calcium channel blockers, terazosin, and doxazosin were allowed for uncontrolled hypertension. Samples for the PRC and PAC were collected in the morning after patients were out of bed for at least $2 \mathrm{~h}$. The screening test was considered positive when the ARR was $\geq 3.7(\mathrm{ng} / \mathrm{dl}) /(\mu \mathrm{IU} / \mathrm{ml})$.

2.3. Diagnosis. Patients who tested positive or who tested negative but in whom PA was strongly suspected (e.g., recurrent hypokalemia and resistant hypertension) underwent the confirmatory tests (saline infusion test, captopril challenge test, or fludrocortisone suppression test). For the remaining patients who screened negative, one of every three consecutive patients underwent the confirmatory tests, which were performed on three separate days.

The saline infusion test was conducted as follows: patients stayed in the recumbent position for at least $1 \mathrm{~h}$ before and during the infusion of 2 liters of $0.9 \%$ saline over $4 \mathrm{~h}$, starting at $8 \mathrm{a} . \mathrm{m}$. Blood samples to measure the PRC, PAC, cortisol, and plasma potassium were drawn at time zero and after $4 \mathrm{~h}$; during the test, patients fasted, and their blood pressure and heart rate were strictly monitored.

The captopril challenge test was conducted as follows: patients received $50 \mathrm{mg}$ of oral captopril at 8-9a.m. after sitting or standing for at least $1 \mathrm{~h}$. Blood samples were drawn for measurement of the PRC and PAC at time zero and at $2 \mathrm{~h}$ after the challenge, with patients remaining seated during this period.

The fludrocortisone suppression test was conducted as follows: patients received $0.1 \mathrm{mg}$ of oral fludrocortisone every $6 \mathrm{~h}$ for $4 \mathrm{~d}$, together with slow-release potassium chloride $(\mathrm{KCl})$ supplements to maintain plasma $\mathrm{K}^{+}$levels close to $4.0 \mathrm{mmol} /$ liter, sodium chloride $(\mathrm{NaCl})$ supplements (30 mmol thrice daily with meals), and sufficient dietary salt to maintain a urinary sodium excretion rate of at least $3 \mathrm{mmol} / \mathrm{kg}$. On day four, the PAC and PRC were measured at 10 a.m. with the patient in a seated posture and the plasma cortisol was measured at 7 a.m. and 10 a.m.

The diagnosis of PA was established according to the fludrocortisone suppression test criteria $[19,20]$ : when the PAC was not suppressed to less than $8 \mathrm{ng} / \mathrm{dl}(220 \mathrm{pmol} / \mathrm{l})$ on day four, the diagnosis of PA was considered. In addition, if adrenal venous sampling (AVS) was performed despite a negative FST and demonstrated lateralization that led to unilateral adrenalectomy, these patients were included in the PA group.

For patients who tested positive during screening but did not undergo the fludrocortisone suppression test, if the postinfusion PAC was over $8 \mathrm{ng} / \mathrm{dl}$ during the saline infusion test or the PAC suppression ratio was higher than $11 \mathrm{ng} / \mathrm{dl}$ during the captopril challenge test, PA was considered [18]. For subtyping, the diagnosis of aldosterone-producing adenoma 


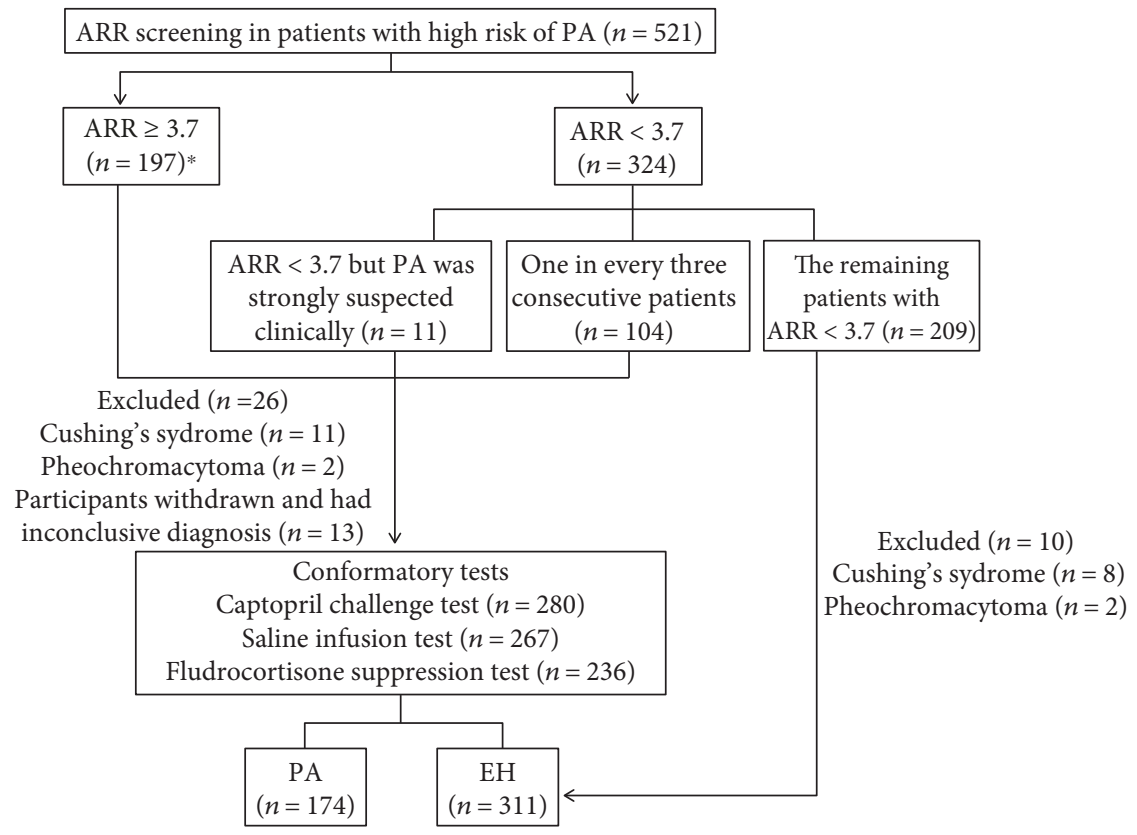

Figure 1: Flow of participants. *Six patients with spontaneous hypokalemia, undetectable renin, and PAC $>20$ ng/dl, though no further confirmatory testing was performed, were included as PA.

required fulfillment of all of the following criteria: [1] unilateral excessive aldosterone secretion confirmed by AVS and/or evidence of a unilateral adrenocortical nodule on CT scan, [2] pathologically confirmed adenoma after surgery, and [3] complete biochemical success during the postadrenalectomy follow-up [21].

In patients with confirmed PA onset at younger age (earlier than 20 years) and in those who had a family history of PA or of strokes at a young age, chimeric CYP11B1/ CYP11B2 genetic testing for glucocorticoid remediable aldosteronism was performed with a long polymerase chain reaction technique [22]. A dexamethasone suppression test and catecholamine assays were performed to rule out hypercortisolism and pheochromocytoma. The flow of participants was shown in Figure 1.

2.4. Biochemical Measurements. The PRC and PAC were measured with an automated chemiluminescence immunoassay (LIAISON; DiaSorin, Italy). The analytical sensitivity (defined as the minimum detectable dose that could be distinguished from zero) for the PRC was $0.53 \mu \mathrm{IU} / \mathrm{ml}$, and the functional sensitivity (defined as the concentration at which the between-assay coefficient of variation exceeded $20 \%$ ) for the PRC was $1.6 \mu \mathrm{IU} / \mathrm{ml}$. The intra-assay coefficient of variations (repeatability) and interassay coefficient of variations (reproducibility) for the PRC ranged from 1.2\% to $3.7 \%$ and $2.9 \%$ to $12.8 \%$, respectively. The PAC assay is capable of measuring a range from $2.2 \mathrm{ng} / \mathrm{dl}$ (analytical sensitivity) to $100 \mathrm{ng} / \mathrm{dl}$, with a functional sensitivity of $3 \mathrm{ng} / \mathrm{dl}$. The intra-assay coefficient of variations for the PAC ranged from $2.4 \%$ to $4.8 \%$. The interassay coefficient of variations ranged from $4.4 \%$ to $6.7 \%$. Quality control was performed every day in the laboratory.
2.5. Statistical Analysis. SPSS 21 was used for statistical analysis. The results below the detection level were set to each assay's respective analytical sensitivity value for comparative purposes. The distribution of the data was analyzed with the Kolmogorov-Smirnov test. Normally distributed variables were expressed as the mean \pm standard deviation (SD); variables with a skewed distribution were expressed as the median (quartile range); categorical variables were described as percentages. Variables with a skewed distribution were analyzed after a natural logarithm transformation. Associations between age and the PRA, PAC, and ARR were calculated with Spearman correlations. One-way ANOVA was used to analyze the trends of the PRA, PAC, and ARR with changes in age. Categorical variables were analyzed with $\chi 2$ test, and quantitative variables were analyzed with Student's $t$-test. To assess the diagnostic accuracy of the screening tests, parameters including the sensitivity and specificity were calculated. $P$ values $<0.05$ (two-tailed) were considered statistically significant.

\section{Results}

3.1. Clinical Characteristics of the Subjects. Five hundred and twenty-one hypertensive patients were screened for PA. Thirty-six patients were excluded from the analysis: 23 patients diagnosed with other causes of secondary hypertension and 13 patients with inconclusive diagnoses. Six patients with spontaneous hypokalemia, undetectable renin concentrations, and $\mathrm{PAC}>20 \mathrm{ng} / \mathrm{dl}$ were included as PA, even though no further confirmatory testing was performed. Overall, 174 patients diagnosed with PA and 311 patients diagnosed with essential hypertension (EH) were included in the analysis (Figure 1). 
TABLE 1: Characteristics of patients with PA and EH.

\begin{tabular}{|c|c|c|c|}
\hline & $\begin{array}{l}\text { Essential hypertension } \\
(n=311)\end{array}$ & $\begin{array}{l}\text { Primary aldosteronism } \\
(n=174)\end{array}$ & $P$ value \\
\hline Gender (M/F) & $149 / 162$ & $82 / 92$ & 0.925 \\
\hline $\operatorname{APA}(n, \%)$ & - & $92,51.7$ & - \\
\hline Age (years) & $47.77 \pm 14.88$ & $48.98 \pm 12.41$ & 0.364 \\
\hline Duration of HT (years) & $3(1,7)$ & $7(2,12)^{*}$ & 0.042 \\
\hline History of diabetes $(n)$ & 72 & 23 & 0.009 \\
\hline History of CVD $(n)$ & 18 & 23 & 0.006 \\
\hline BMI $\left(\mathrm{kg} / \mathrm{m}^{2}\right)$ & $25.09(22.69,27.7)$ & $24.36(22.3,26.72)$ & 0.024 \\
\hline SBP $(\mathrm{mmHg})$ & $152.99 \pm 21.54$ & $157.10 \pm 20.64$ & 0.042 \\
\hline $\mathrm{DBP}$ (mmHg) & $93.69 \pm 15.63$ & $96.50 \pm 15.38$ & 0.047 \\
\hline Serum $\mathrm{K}^{+}(\mathrm{mmol} / \mathrm{l})$ & $4.0(3.8,4.2)$ & $3.2(2.7,3.7)$ & $<0.001$ \\
\hline Serum $\mathrm{Na}^{+}(\mathrm{mmol} / \mathrm{l})$ & $142(140,143)$ & $143(141,145)$ & $<0.001$ \\
\hline $24 \mathrm{~h}$ Urinary $\mathrm{Na}^{+}(\mathrm{mmol})$ & $140.6(98.775,204.75)$ & $153.15(112.48,208.95)$ & 0.244 \\
\hline $24 \mathrm{~h}$ Urinary $\mathrm{K}^{+}(\mathrm{mmol})$ & $33.7(25.35,44.65)$ & $50.35(34.68,68.65)$ & $<0.001$ \\
\hline PAC (ng/dl) & $11.00(6.93,17.20)$ & $29.30(20.20,53.08)$ & $<0.001$ \\
\hline $\operatorname{PRC}(\mu \mathrm{IU} / \mathrm{ml})$ & $17.20(7.00,35.40)$ & $1.95(0.65,4.85)$ & $<0.001$ \\
\hline ARR & $0.63(0.29,1.47)$ & $17.19(5.34,54.67)$ & $<0.001$ \\
\hline
\end{tabular}

Data were expressed as mean $\pm \mathrm{SD}, \%$, or median (interquartile range). APA: aldosterone producing adenoma; HT: hypertension; CVD: cardiovascular disease; BMI: body mass index; SBP: systolic blood pressure; DBP: diastolic blood pressure; serum $\mathrm{K}^{+}$: concentration of serum potassium; serum $\mathrm{Na}^{+}$: concentration of serum sodium; PAC: plasma aldosterone concentration; PRC: plasma renin concentration.

The clinical characteristics of the subjects are shown in Table 1. There were no significant differences between the $\mathrm{EH}$ and PA groups in terms of gender or age. Patients with PA had higher levels of SBP, DBP, PAC, ARR, serum sodium, and $24 \mathrm{~h}$ urine potassium, whereas those with PA had lower serum potassium and PRC levels.

The PRC and PAC were negatively associated with age ( $r=-0.46, P<0.001$ and $r=-0.19, P<0.001)$, whereas the ARR was positively associated with age $(r=0.35, P<0.001)$ in the EH group (Figures 2(a)-2(c)). In the PA group, the PRC was negatively associated with age $(r=-0.18, P=$ $0.016)$, while neither the PAC $(r=-0.10, P=0.18)$ nor the ARR $(r=0.10, P=0.20)$ were significantly associated with age (Figures $2(\mathrm{~d})-2(\mathrm{f}))$.

To analyze the tendency of changes in the PAC, PRA, and ARR in different age groups, subjects were subdivided into four groups as follows: $<40,40-49,50-59$, and $\geq 60$ years old. The clinical characteristics of the subjects in the different age subgroups are shown in Table 2. In the EH group, both the PAC $(P$ for trend $=0.001)$ and PRC $(P$ for trend $<0.001)$ significantly declined with increasing age. Notably, the ARRs gradually increased with increasing age $(P$ for trend $<0.001)$. In the PA group, the PRC significantly declined with increasing age $(P$ for trend $=0.01)$, but the trends for the PAC and ARR were not significant $(P$ for trend $=0.442$ and 0.183 , resp.) (Figure 3).

In the $\mathrm{EH}$ group, the proportion of patients with a positive ARR (ARR $\geq 3.7(\mathrm{ng} / \mathrm{dl}) /(\mu \mathrm{IU} / \mathrm{ml}))$ was much higher in patients $\geq 60$ years old than in younger patients $(P=0.01$ versus $<40$ years, $P=0.006$ versus $40-49$ years, $P=0.014$ versus 50-59 years) (Figure 4).
3.2. Cutoffs of the ARR for PA Screening. An ARR of $3.7(\mathrm{ng} / \mathrm{dl}) /(\mu \mathrm{IU} / \mathrm{ml})$ showed a sensitivity of $90.7 \%$ and a specificity of $85.6 \%$ in the whole cohort. However, the accuracy of the ARR varied greatly in the different age groups (Table 3$)$. An ARR of $3.7(\mathrm{ng} / \mathrm{dl}) /(\mu \mathrm{IU} / \mathrm{ml})$ showed a sensitivity of $100 \%$ and a specificity of $80 \%$ in patients older than 60 years. However, with this cutoff, 10 out of 56 (18\%) PA patients aged 40-49 and 5 out of 39 (13\%) PA patients aged 50-59 were misdiagnosed as EH patients. Using an ARR of 3.7 as the cutoff value, the highest rate of misdiagnosis was found in PA patients younger than 40, meaning that 10 out of 39 (26\%) PA patients were missed. As the ARR was used as a screening index, high sensitivity was a priority. To achieve a sensitivity higher than $90 \%$, the optimal ARR cutoff needed to be lowered to $2.0(\mathrm{ng} / \mathrm{dl}) /$ $(\mu \mathrm{IU} / \mathrm{ml})$ for patients 40-49 years old and for patients 50-59 years old. With this cutoff value, the sensitivity and specificity in patients 40-49 years old were similar to those in patients 50-59 years old. The sensitivity was only $79 \%$ in patients younger than 40 years old, although the ARR was as low as $2.0(\mathrm{ng} / \mathrm{dl}) /(\mu \mathrm{IU} / \mathrm{ml})$. To achieve a sensitivity higher than $90 \%$, the optimal ARR cutoff value needed to be lowered to $1.0(\mathrm{ng} / \mathrm{dl}) /(\mu \mathrm{IU} / \mathrm{ml})$ for patients younger than 40 years old. Of note, in the patients older than 60 years, ARR values of 1.0 and 2.0 resulted in low specificities of $42 \%$ and $58 \%$, respectively.

\section{Discussion}

The present study included a relatively large number of patients to establish age-related ARR cutoffs based on the 
EH

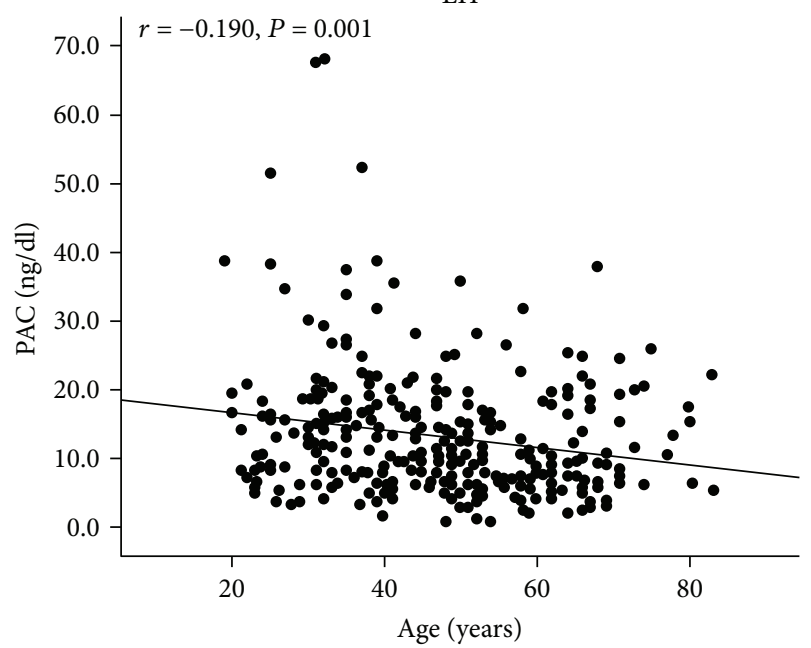

(a)

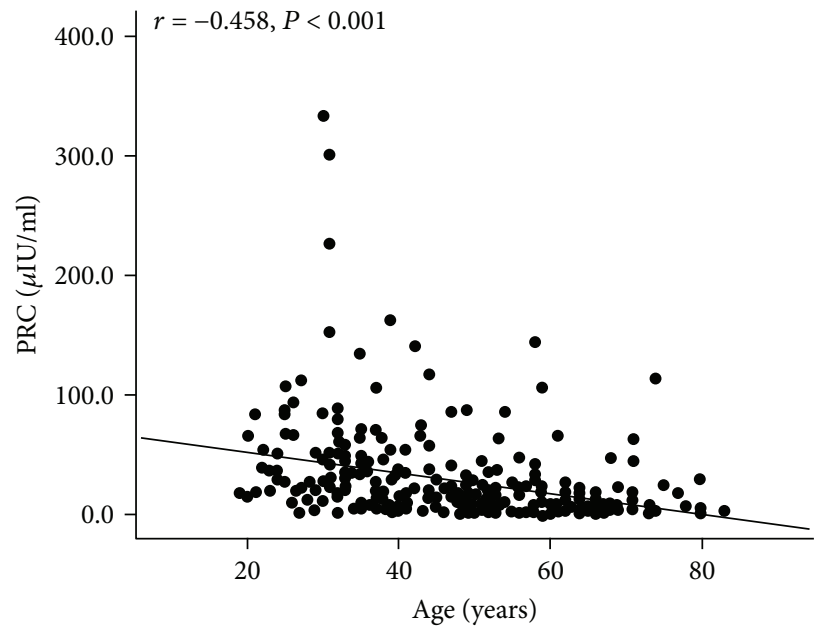

(b)

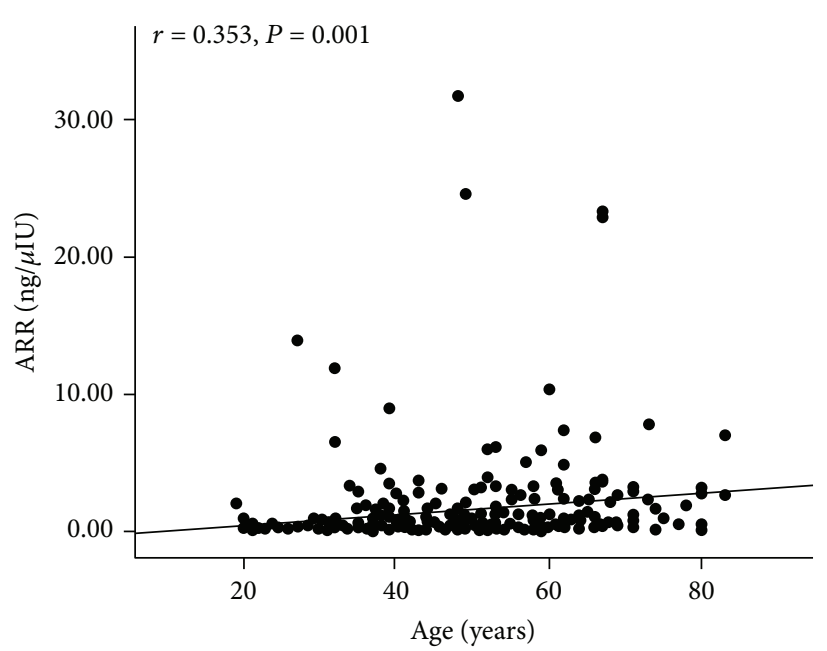

(c)

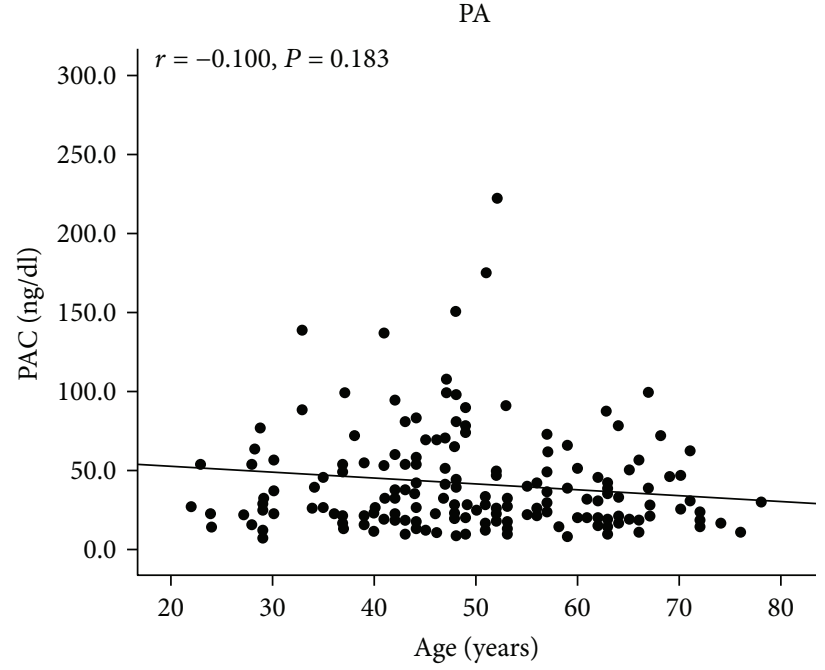

(d)

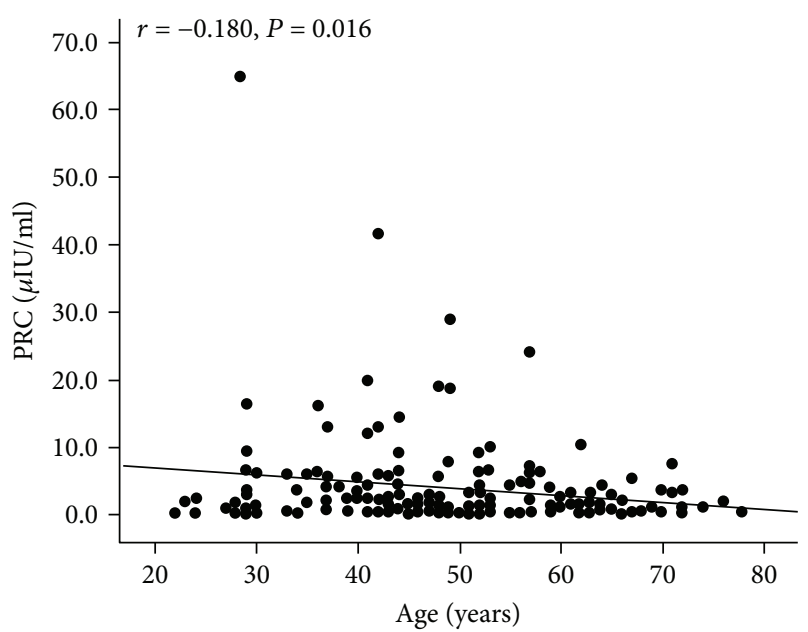

(e)

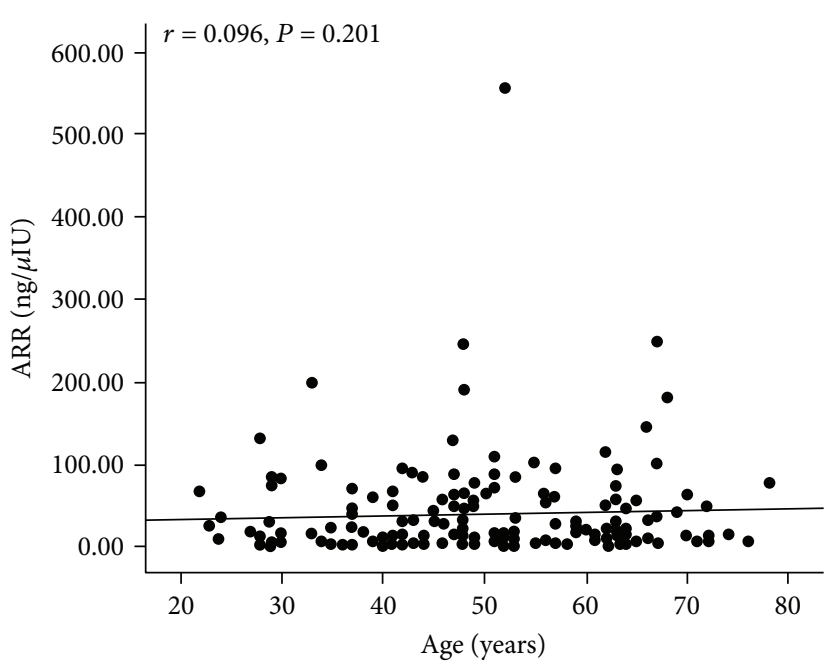

(f)

FIGURE 2: Scatter plots showing the correlation of PRC, PAC, and ARR levels with age in patients with EH and PA. The PAC (a) and PRC (b) were negatively correlated with age whereas ARR (c) was positively correlated with age in patients with EH. In the PA group, PRC was negatively correlated with age but neither PAC nor ARR was significantly associated with age (d-f). 


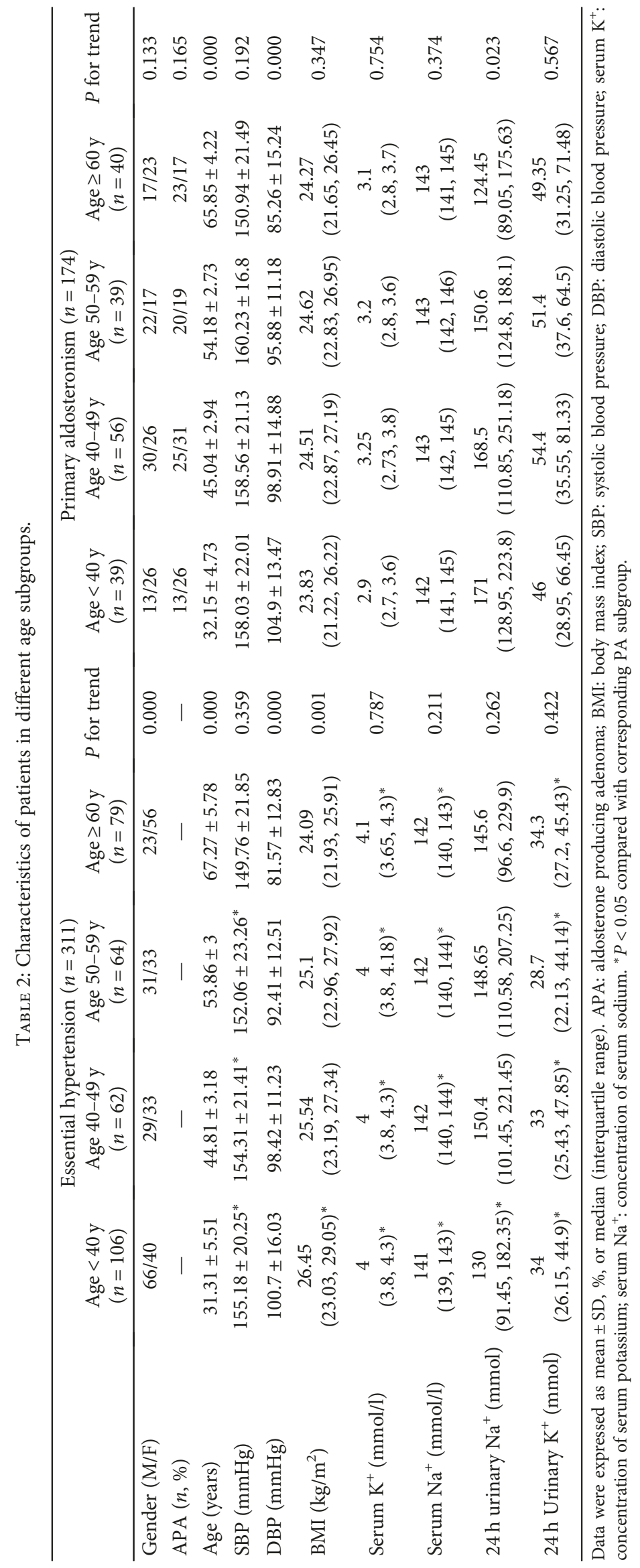




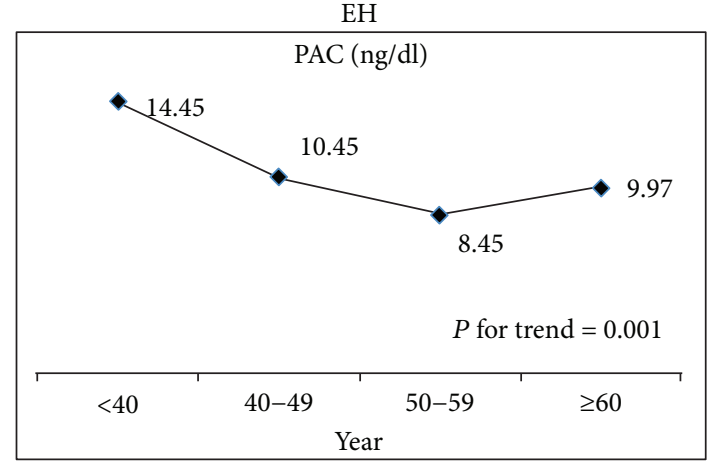

(a)

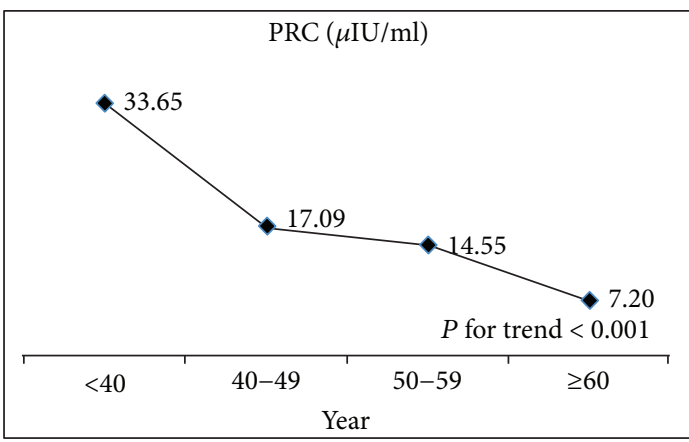

(b)

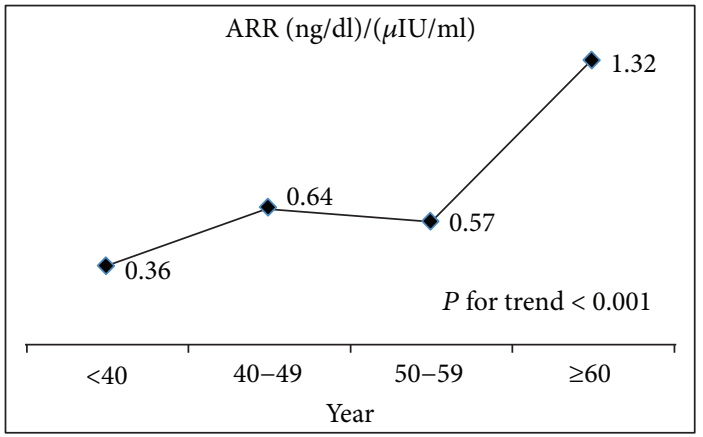

(c)

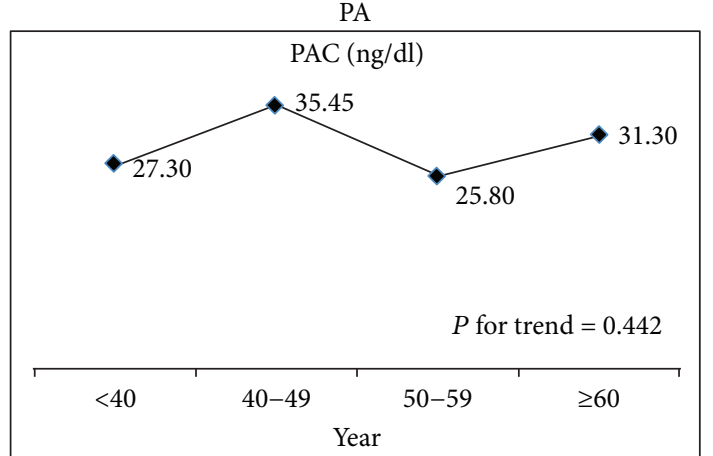

(d)

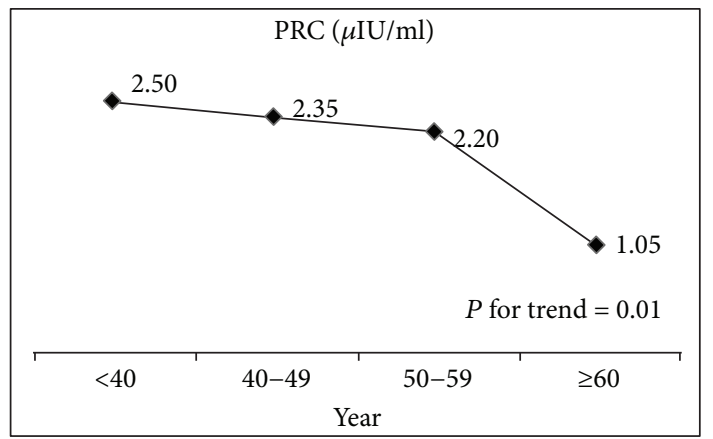

(e)

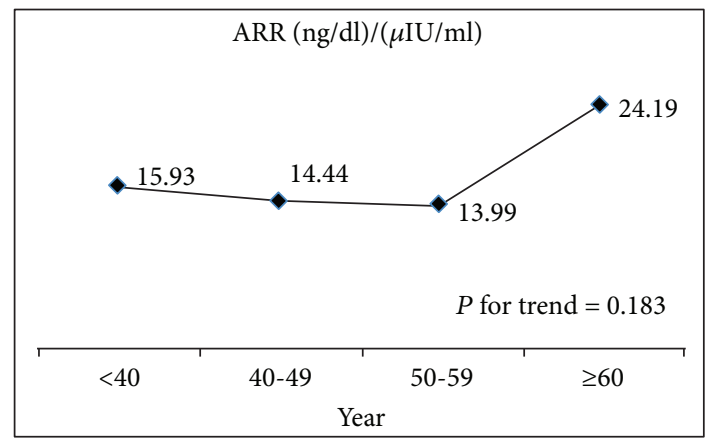

(f)

FIgURE 3: Line chart of PAC, PRC, and ARR in different age groups in patients with EH and PA. In the EH group, PRA lowered more than PAC and led to higher ARR with increasing age. In the PA group, PRA significantly declined with increasing age, but the trends for PAC and ARR were not significant. ARR: aldosterone to renin ratio; PA: primary aldosteronism; PAC: plasma aldosterone concentration; PRC: plasma renin concentration.

PAC and PRC, which provide valuable information for PA screening. Our study revealed that using the ARR cutoff value of $3.7(\mathrm{ng} / \mathrm{dl}) /(\mu \mathrm{IU} / \mathrm{ml})$ was associated with a high risk of false negative results in hypertensive patients, particularly in those younger than 60 years. An ARR of $3.7(\mathrm{ng} / \mathrm{dl}) /$ $(\mu \mathrm{IU} / \mathrm{ml})$ is optimal for hypertensive patients older than 60 years, while for patients 40-59 years old, the optimal ARR cutoff value is $2.0(\mathrm{ng} / \mathrm{dl}) /(\mu \mathrm{IU} / \mathrm{ml})$, and for those younger than 40 years, an ARR of $1.0(\mathrm{ng} / \mathrm{dl}) /(\mu \mathrm{IU} / \mathrm{ml})$ may be more reasonable.

In our study, when using the ARR cutoff of 3.7 (ng/dl)/ ( $\mu \mathrm{IU} / \mathrm{ml})$ for PA screening in the whole cohort, the sensitivity was 90\%, which is in accordance with previous reports. In the study conducted by Burrello et al. [5], which included $75 \mathrm{EH}$ patients and $20 \mathrm{PA}$ patients, ARRs of $3.7,2.7$, and $1.0(\mathrm{ng} / \mathrm{dl}) /(\mu \mathrm{IU} / \mathrm{ml})$ were reported to have sensitivities of $90 \%, 95 \%$, and $100 \%$, respectively. However, the study did not perform an age subgroup analysis due to the small sample size. Since case detection requires high sensitivity, the authors suggest an ARR between 1.0 and $2.7(\mathrm{ng} / \mathrm{dl}) /(\mu \mathrm{IU} / \mathrm{ml})$ for PA screening. However, our data showed that a low ARR cutoff (e.g., 1-2.4 (ng/dl)/( $\mu \mathrm{IU} / \mathrm{ml})$ ) caused a high false positive rate $(42 \%-63 \%)$ and led to unnecessary confirmatory testing, which carries the potential risk for acute volume overload in elderly patients (i.e., $\geq 60$ years).

Several studies have focused on the effects of age on ARR screening based on the PRA but have drawn inconsistent conclusions. In a study conducted by Yin et al. [15], 216 subjects with PA and 657 subjects with non-PA were included. Similar to our results, the study found that changes in the 


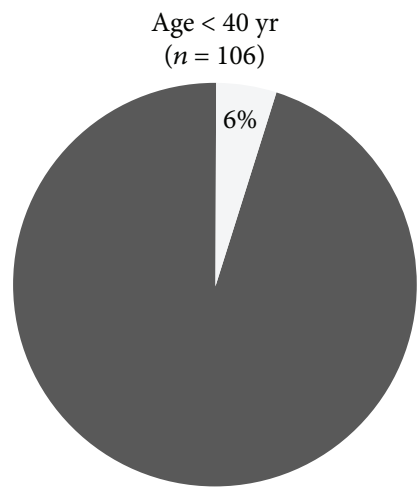

ARR $\geq 3.7$

ARR $<3.7$

(a)

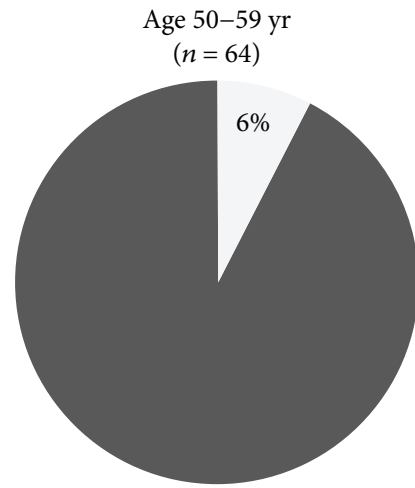

ARR $\geq 3.7$

ARR $<3.7$

(c)

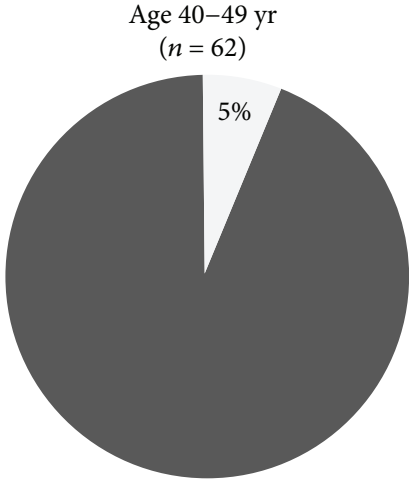

ARR $\geq 3.7$

ARR $<3.7$

(b)

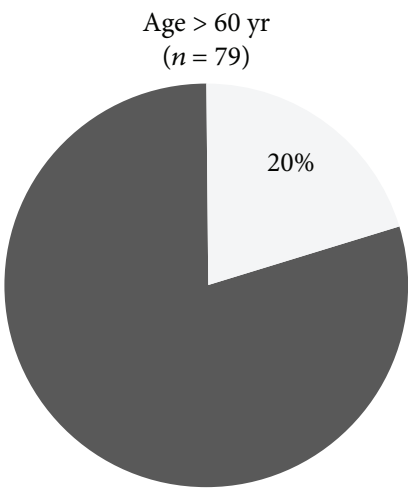

ARR $\geq 3.7$

$A R R<3.7$

(d)

FIGURE 4: Pie charts showing the proportion of $\mathrm{EH}$ patients with positive or negative ARR in different age subgroups.

TABle 3: Accuracy of ARR for PA screening in different age subgroups.

\begin{tabular}{|c|c|c|c|c|c|c|c|c|}
\hline \multirow{2}{*}{$\begin{array}{l}\text { Cutoffs } \\
\operatorname{ARR}(\mathrm{ng} / \mathrm{dl}) /(\mu \mathrm{IU} / \mathrm{ml})\end{array}$} & \multicolumn{2}{|c|}{$\begin{array}{c}\text { Age }<40 \text { y } \\
(n=145)\end{array}$} & \multicolumn{2}{|c|}{$\begin{array}{c}\text { Age } 40-49 y \\
(n=118)\end{array}$} & \multicolumn{2}{|c|}{$\begin{array}{c}\text { Age } 50-59 y \\
(n=103)\end{array}$} & \multicolumn{2}{|c|}{$\begin{array}{c}\text { Age } \geq 60 y \\
(n=119)\end{array}$} \\
\hline & Sen & Spe & Sen & Spe & Sen & Spe & Sen & Spe \\
\hline 1 & 0.90 & 0.82 & 0.95 & 0.69 & 0.97 & 0.59 & 1.00 & 0.42 \\
\hline 1.5 & 0.85 & 0.88 & 0.90 & 0.81 & 0.95 & 0.75 & 1.00 & 0.54 \\
\hline 2 & 0.79 & 0.91 & 0.90 & 0.84 & 0.95 & 0.80 & 1.00 & 0.58 \\
\hline 2.4 & 0.77 & 0.92 & 0.86 & 0.89 & 0.89 & 0.80 & 1.00 & 0.63 \\
\hline 3.7 & 0.74 & 0.94 & 0.82 & 0.95 & 0.87 & 0.94 & 1.00 & 0.80 \\
\hline
\end{tabular}

plasma renin and ARR were more obvious in the non-PA group compared with the PA group. This phenomenon might be explained by that the effects of age on the PAC and ARR might be weakened in PA patients due to the abnormal secretion of PAC and suppression of PRC. In a study by Yin et al. [15], the subjects were divided into four age groups ( $\leq 39,40-49,50-59$, and $\geq 60$ years old) and the authors suggested that the criteria for the ARR needed to be set higher in the older population, but they did not recommend specific ARR cutoffs for each group. A study by Luo et al. [16] included $13 \mathrm{PA}$ and $69 \mathrm{EH}$ patients older than 65 years old and $32 \mathrm{PA}$ and $41 \mathrm{EH}$ patients younger than 65 years old. The author recommended an ARR cutoff value of 556 (PAC as pmol/l and PRA as $\mathrm{ng} / \mathrm{ml} / \mathrm{h}$ ) in the elderly and an ARR cutoff value of 272 in the nonelderly. Contrary to these studies, Unger et al. [14] reported that although the number of $\mathrm{EH}$ patients with elevated ARR increased with advancing age, the ARR cutoffs for PA screening were not affected by age. However, when they analyzed the accuracy of the ARR in different age subgroups, only EH patients were 
divided according to age, while the 39 PA patients were not divided accordingly.

Our results must be interpreted in the context of the strengths and drawbacks of the study. First, although an $A R R \geq 3.7$ was used as the positive cutoff in the screening protocol, many patients who tested negative also underwent the confirmatory tests, which avoided overestimating the accuracy of the ARR. Furthermore, the data were derived from a prospectively designed study and the procedures of screening and confirmation were standardized according to the guidelines. Some limitations of the study are worth mentioning. This study was carried out in a single tertiary hospital center, and the PA prevalence was higher than that in the general population in which the tests will be applied to. Nevertheless, a single-center study leads to better standardization of tests and better quality control. In addition, the study was retrospective and the PRA results were not available; therefore, we could not compare "PRC-based ARR" with "PRA-based ARR". Based on the study protocol, 209 patients with an $\mathrm{ARR}<3.7$ who did not have any confirmatory test performed were included as EH patients. However, as our published results showed, 24 out of 135 patients (18\%) with a final diagnosis of PA had an ARR $<3.7$ [18]. Therefore, a small portion of the 209 patients who did not perform any confirmatory test might be misdiagnosed as $\mathrm{EH}$ patients, which might lead to an overestimation of the sensitivity of the 3.7 cut-off.

4.1. Perspectives. In summary, when an automated chemiluminescent immunoassay is used, an ARR of 3.7 (ng/dl)/ $(\mu \mathrm{IU} / \mathrm{ml})$ is associated with a high risk of false negative results in patients younger than 60 years old. An ARR of $3.7(\mathrm{ng} / \mathrm{dl}) /(\mu \mathrm{IU} / \mathrm{ml})$ is optimal for hypertensive patients older than 60 years old; for patients 40-59 years old, the optimal ARR cutoff is $2.0(\mathrm{ng} / \mathrm{dl}) /(\mu \mathrm{IU} / \mathrm{ml})$; and for those younger than 40 years old, an ARR of $1.0(\mathrm{ng} / \mathrm{dl}) /(\mu \mathrm{IU} / \mathrm{ml})$ might be more reasonable.

\section{Disclosure}

Ying Song is the co-first author.

\section{Conflicts of Interest}

The authors declare that they have no conflicts of interest.

\section{Acknowledgments}

This study is funded by the National Key Clinical Specialties Construction Program of China to the Department of Endocrinology, the First Affiliated Hospital of Chongqing Medical University, and the National Natural Science Foundation of China (81670785) (to Qifu Li) and the Fundamental Science and Advanced Technology Research of Chongqing (Major Project, cstc2015jcyjBX0096) (to Qifu Li) and Chongqing Science and Technology Committee Innovation Project (Technology Development and Application of Precision Medicine, cstc2016shms-ztzx1003) (to Qifu Li) and Medical Research Program of Health and Family Planning
Commission of Chongqing Municipality (2016MSXM004) (to Shumin Yang). The authors thank the Laboratory of Endocrine and Laboratory of Lipid and Glucose Metabolism, the First Affiliated Hospital of Chongqing Medical University. The authors also thank Richa Goswami, Changhong Zhao, Zhengping Feng, Rong Li, Huacong Deng, Chun Liu, Bo Zhou, Wei Ren, Jian Long, Lilin Gong (from Department of Endocrinology, the First Affiliated Hospital of Chongqing Medical University), Chuan Peng, Rufei Gao, and Xiaoqiu Xiao (from Laboratory of Lipid and Glucose Metabolism, the First Affiliated Hospital of Chongqing Medical University) for suggestions on the study design and revision. The members of the Chongqing Primary Aldosteronism Study (CONPASS) group are Suxin Luo, MD, PhD; Kangla Liao, $\mathrm{MD}$; Yao Zhang, MD, PhD; Yunfeng He, MD, PhD; Ming Xiao, PhD; and Bin Peng, PhD.

\section{References}

[1] J. W. Funder, R. M. Carey, F. Mantero et al., “The management of primary aldosteronism: case detection, diagnosis, and treatment: an Endocrine Society clinical practice guideline," The Journal of Clinical Endocrinology and Metabolism, vol. 101, no. 5, pp. 1889-1916, 2016.

[2] D. Denimal and L. Duvillard, "2016 Endocrine Society guidelines update for the diagnosis of primary aldosteronism: are the proposed aldosterone-to-renin ratio cut-off values relevant in the era of fully automated immunoassays?," Annals of Clinical Biochemistry, vol. 53, no. 6, pp. 714-715, 2016.

[3] F. H. Perschel, R. Schemer, L. Seiler et al., "Rapid screening test for primary hyperaldosteronism: ratio of plasma aldosterone to renin concentration determined by fully automated chemiluminescence immunoassays," Clinical Chemistry, vol. 50, no. 9, pp. 1650-1655, 2004.

[4] C. A. Dorrian, B. J. Toole, S. Alvarez-Madrazo, A. Kelly, J. M. C. Connell, and A. M. Wallace, "A screening procedure for primary aldosteronism based on the Diasorin Liaison ${ }^{\circledR}$ automated chemiluminescent immunoassay for direct renin," Annals of Clinical Biochemistry, vol. 47, no. 3, pp. 195-199, 2010.

[5] J. Burrello, S. Monticone, F. Buffolo et al., "Diagnostic accuracy of aldosterone and renin measurement by chemiluminescent immunoassay and radioimmunoassay in primary aldosteronism," Journal of Hypertension, vol. 34, no. 5, pp. 920927, 2016.

[6] N. Belaidi, A. Georges, J. Brossaud, and J. B. Corcuff, "Aldosterone determination: comparison of a RIA assay and a CLIA assay," Clinical Biochemistry, vol. 48, no. 1-2, pp. 89-92, 2015.

[7] C. Lonati, N. Bassani, A. Gritti, E. Biganzoli, and A. Morganti, "Measurement of plasma renin concentration instead of plasma renin activity decreases the positive aldosteroneto-renin ratio tests in treated patients with essential hypertension," Journal of Hypertension, vol. 32, no. 3, pp. 627-634, 2014.

[8] G. P. Rossi, M. Barisa, A. Belfiore et al., "The aldosterone-renin ratio based on the plasma renin activity and the direct renin assay for diagnosing aldosterone-producing adenoma," Journal of Hypertension, vol. 28, no. 9, pp. 1892-1899, 2010.

[9] X. Li, R. Goswami, S. Yang, and Q. Li, "Aldosterone/direct renin concentration ratio as a screening test for primary 
aldosteronism: a meta-analysis," Journal of the ReninAngiotensin-Aldosterone System, vol. 17, no. 3, 2016.

[10] P. Ferrari, S. G. Shaw, J. Nicod, E. Saner, and J. Nussberger, "Active renin versus plasma renin activity to define aldosterone-to-renin ratio for primary aldosteronism," Journal of Hypertension, vol. 22, no. 2, pp. 377-381, 2004.

[11] P. Glinicki, W. Jeske, L. Bednarek-Papierska et al., "The ratios of aldosterone / plasma renin activity (ARR) versus aldosterone / direct renin concentration (ADRR)," Journal of the Renin-Angiotensin-Aldosterone System, vol. 16, no. 4, pp. 1298-1305, 2015.

[12] F. Corbin, P. Douville, and M. Lebel, "Active renin mass concentration to determine aldosterone-to-renin ratio in screening for primary aldosteronism," International Journal of Nephrology and Renovascular Disease, vol. 4, pp. 115120, 2011.

[13] E. Fischer, S. Reuschl, M. Quinkler et al., "Assay characteristics influence the aldosterone to renin ratio as a screening tool for primary aldosteronism: results of the German Conn's registry," Hormone and Metabolic Research, vol. 45, no. 7, pp. 526-531, 2013.

[14] N. Unger, I. Lopez Schmidt, C. Pitt et al., "Comparison of active renin concentration and plasma renin activity for the diagnosis of primary hyperaldosteronism in patients with an adrenal mass," European Journal of Endocrinology, vol. 150, no. 4, pp. 517-523, 2004.

[15] G. Yin, S. Zhang, L. Yan et al., "Effect of age on aldosterone/ renin ratio (ARR) and comparison of screening accuracy of ARR plus elevated serum aldosterone concentration for primary aldosteronism screening in different age groups," Endocrine, vol. 42, no. 1, pp. 182-189, 2012.

[16] Q. Luo, N. F. Li, X. G. Yao et al., "Potential effects of age on screening for primary aldosteronism," Journal of Human Hypertension, vol. 30, no. 1, pp. 53-61, 2016.

[17] C. Nakama, K. Kamide, T. Kawai et al., "The influence of aging on the diagnosis of primary aldosteronism," Hypertension Research, vol. 37, no. 12, pp. 1062-1067, 2014.

[18] Y. Song, S. Yang, W. He et al., "Confirmatory tests for the diagnosis of primary aldosteronism: a prospective diagnostic accuracy study," Hypertension, vol. 71, no. 1, pp. 118124, 2018

[19] M. Stowasser and R. D. Gordon, "Primary aldosteronism: changing definitions and new concepts of physiology and pathophysiology both inside and outside the kidney," Physiological Reviews, vol. 96, no. 4, pp. 1327-1384, 2016.

[20] C. Westerdahl, A. Bergenfelz, J. Larsson et al., "Re-evaluation of the fludrocortisone test: duration, $\mathrm{NaCl}$ supplementation and cut-off limits for aldosterone," Scandinavian Journal of Clinical and Laboratory Investigation, vol. 69, no. 2, pp. 234241, 2009.

[21] T. A. Williams, J. W. M. Lenders, P. Mulatero et al., "Outcomes after adrenalectomy for unilateral primary aldosteronism: an international consensus on outcome measures and analysis of remission rates in an international cohort," The Lancet Diabetes and Endocrinology, vol. 5, no. 9, pp. 689-699, 2017.

[22] I. S. Lee, S. Y. Kim, H. W. Jang et al., "Genetic analyses of the chimeric CYP11B1/CYP11B2 gene in a Korean family with glucocorticoid-remediable aldosteronism," Journal of Korean Medical Science, vol. 25, no. 9, pp. 1379-1383, 2010. 


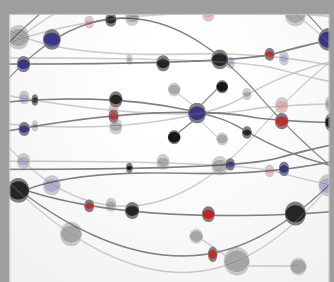

The Scientific World Journal
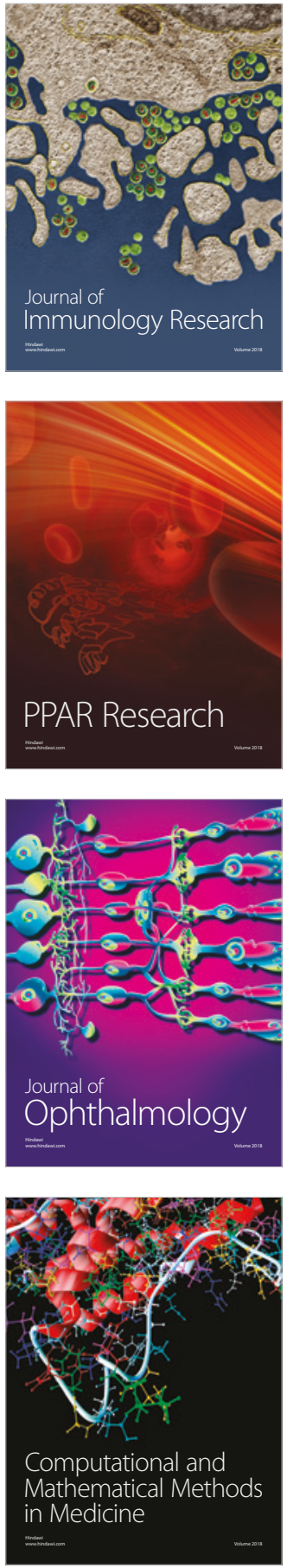

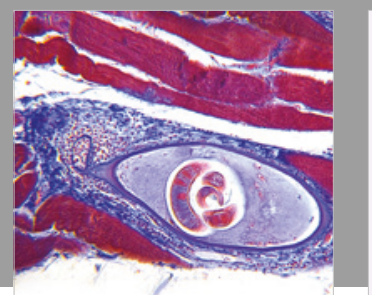

Gastroenterology Research and Practice

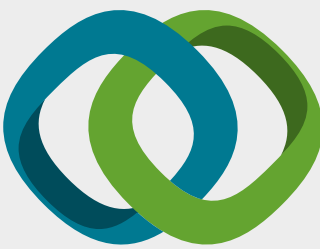

\section{Hindawi}

Submit your manuscripts at

www.hindawi.com
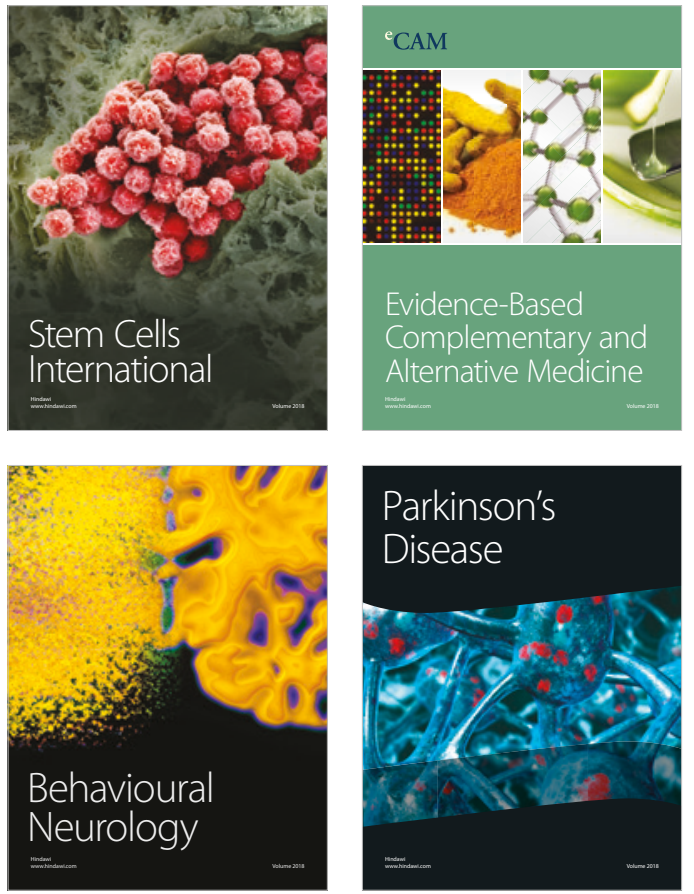

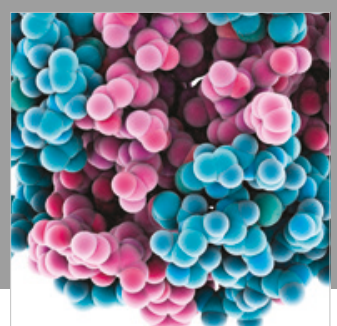

ournal of

Diabetes Research

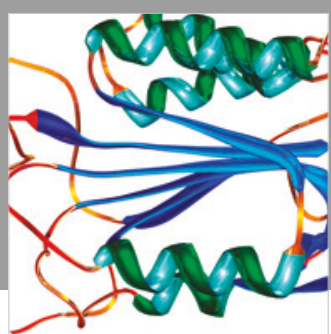

Disease Markers
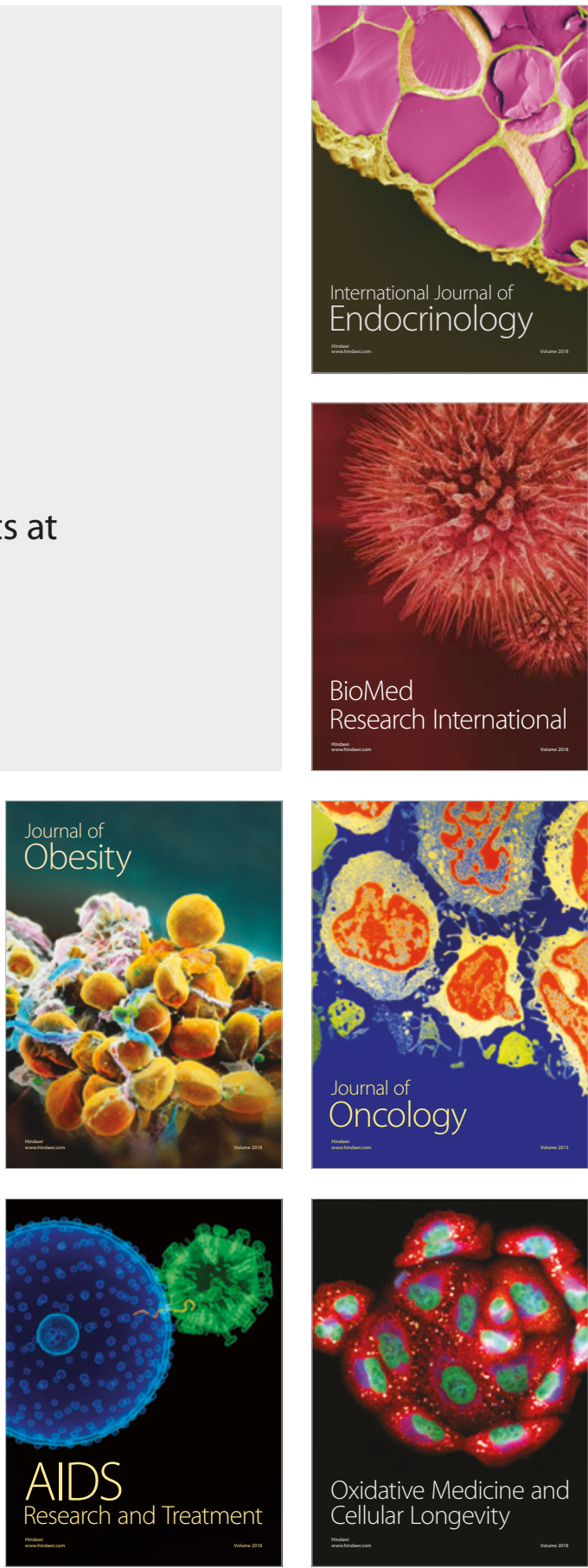